\title{
À propos de la complexité des révoltes dans les
} pays arabes

On the Complexity of the Revolts in the Arab Countries

\section{Nizar MESSARI}

\section{OpenEdition}

12 Journals

\section{Édition électronique}

URL : http://journals.openedition.org/conflits/18401

DOI : $10.4000 /$ conflits. 18401

ISSN : $1777-5345$

Éditeur :

CCLS - Centre d'études sur les conflits lilberté et sécurité, L'Harmattan

\section{Édition imprimée}

Date de publication : 25 juin 2012

Pagination : 187-212

ISBN : 978-2-296-99408-9

ISSN : 1157-996X

Référence électronique

Nizar MESSARI, «À propos de la complexité des révoltes dans les pays arabes », Cultures \& Conflits [En ligne], 85-86 | Printemps-Été 2012, mis en ligne le 25 juin 2013, consulté le 30 mars 2021. URL: http://journals.openedition.org/conflits/18401 ; DOI : https://doi.org/10.4000/conflits.18401 


\section{À propos de la complexité des révoltes dans les pays arabes 1}

\section{Nizar MESSARI}

Nizar Messari est professeur de relations internationales à l'Université Al Akhawayn, au Maroc. Ses recherches portent sur la théorie des relations internationales et sur la sécurité internationale.

itôt l'ancien président de Tunisie Ben Ali avait-il été déchu de son fauteuil
par une révolte populaire qu'une question s'est imposée : s'agissait-il d'un événement unique et sans répercussions dans les autres pays arabes ou des événements similaires allaient-ils se produire dans d'autres pays de la région ? En effet, ce n'est certainement pas la première fois qu'un puissant dictateur de la région est déchu par son peuple et que la situation inspire d'autres peuples arabes. En 1979, après la chute du shah d'Iran, un grand allié de l'Occident en général et des États-Unis en particulier, les manifestations s'étaient multipliées dans le monde arabe. Les monarchies arabes du Golfe et d'ailleurs avaient tremblé dans un contexte d'instabilité grandissante, non seulement au Maghreb mais partout ailleurs dans le monde arabo-musulman. Les peuples des autres pays de la région se disaient, en quelque sorte, que si les Iraniens avaient réussi à renverser le tout-puissant shah, en dépit de sa violente et puissante police secrète et de sa richesse pétrolière, et malgré l'appui total des États-Unis, eux aussi pouvaient y arriver ${ }^{2}$. Il n'en fut rien puisque tous les régimes en place réussirent à se maintenir, les quelques évolutions observables n'étant in fine que des changements dans la continuité. On peut citer l'arrivée de Chadli Bendjedid à la présidence de la république en Algérie en février

1. Bien que j'assume pleinement toute la responsabilité de l'argument que je présente ici, je tiens à remercier le travail de recherche entrepris par Iman Moussaoui et Alia Lahlou sans lequel il aurait été difficile de réaliser ce projet.

2. À ce propos, lire Anderson L., "The State in the Middle East and North Africa”, Comparative Politics, 20-1, 1987, pp. 1-18; Kepel G., Jihad-Expansion et déclin de l'islamisme, Paris, Gallimard, 2000, et en particuliers la première partie ; Muñoz G. M., El Estado Árabe - Crisis de legitimidad y la contestación islamista, Madrid, Ediciones Bellaterra, 2000, et en particuliers la seconde partie de l'ouvrage ; Salem M. A., "Arab Schisms in the 1980's: Old Story or New Order”, The World Today, 38-5, 1982, pp. 175-184 ; Zartman I. W., "Foreign Relations of North Africa", Annals of the American Academy of Political and Social Science, 489, 1987, pp. 13-27. 
1979, suite à la mort du président Houari Boumedienne (avant même le succès final de la révolution iranienne) ; ou encore l'accès du vice-président Saddam Hussein à la présidence de la république en Irak en juillet 1979 en remplacement du président Ahmed Hassan al-Bakr, entérinant le statut de celui qui était déjà considéré comme l'homme fort du parti Baas en Irak depuis le coup d'État de 1968. La configuration s'est révélée similaire en Égypte avec l'arrivée au pouvoir du vice-président Hosni Moubarak en octobre 1981 suite à l'assassinat du président Anouar el-Sadat ${ }^{3}$.

Une autre comparaison s'est néanmoins rapidement imposée : en lieu et place de 1979, le modèle à étudier est devenu 1989 et la chute en Europe de l'Est des régimes alliés de l'Union soviétique. Dans le monde arabe, 2011 est désormais mis en parallèle avec l'effondrement $\mathrm{du}$ "bloc » soviétique. Les chutes successives du président Ben Ali et du président Moubarak, suivies de celle du colonel Kadhafi en Lybie laisseraient anticiper celles des présidents Salah au Yémen et al Assad en Syrie, même si les voies de sortie sont clairement différentes. La dynamique sociale qu'il faut désormais comprendre et expliquer est la succession des révoltes dans le monde arabe, ainsi que leurs évolutions ${ }^{4}$.

Je me propose dans cet article d'analyser les bouleversements dans le monde arabe au cours de l'année 2011 à la lumière de quelques modèles théoriques qui visent à expliquer les mobilisations sociales et populaires. Mon propos est d'abord de fournir une clé de compréhension de ces événements, avant d'examiner les amendements que ces mobilisations apportent à ces modèles théoriques. Cet objectif est d'autant plus important que si les signes avantcoureurs de la crise dans les pays arabes étaient abondants, connus et montrés par de nombreux rapports rédigés par le Programme des Nations unies pour le développement (PNUD) ou certaines initiatives européennes et américaines ${ }^{5}$, la force et l'ampleur des révoltes arabes a surpris politiciens et observateurs de la même façon. La question à laquelle je veux répondre ici est donc double : comment comprendre les événements de 2011 et leur ampleur ?

3. Lire Kepel G., Jihad - Expansion et déclin de l'islamisme, op.cit. ; Muñoz, G. M., El Estado Árabe - Crisis de legitimidad y la contestación islamista, op.cit. Pour une discussion axée sur la chute des régimes et les instabilités qui s'en suivent, voir Betts R. K., et Huntington S. P., "Dead Dictators and Rioting Mobs: Does the Demise of Authoritarian Rulers Lead to Political Instability?”, International Security, 10-3, 1985-1986, pp. 112-146, et en particulier le tableau 1, p. 114.

4. Il faut néanmoins réitérer que, jusqu’à présent, un changement de régime effectif n'a eu lieu qu'en Tunisie et en Libye, alors qu'en Égypte, les militaires sont restés aux commandes en dépit de l'éviction du président Moubarak. Il est donc trop tôt pour se prononcer sur la possibilité de changements complets dans d'autres pays en dehors de la Tunisie et du cas légèrement exceptionnel de la Libye.

5. Depuis 2002, le Programme des Nations unies pour le développement (PNUD) a produit une série de rapports sur le développement humain dans le monde arabe, et dont les résultats furent particulièrement alarmants, puisqu'ils situaient celui-ci loin derrière plusieurs autres régions du monde. 


\section{Que nous enseignent les études sur les révoltes et les mobilisations popu- laires ?}

Deux questions distinctes mais liées s'imposent : pourquoi plusieurs peuples arabes se sont-ils soulevés au cours de l'année 2011 ? Pourquoi ces soulèvements ont-ils eu lieu de façon presque simultanée ? D’un point de vue théorique, la littérature existante nous dirige vers deux concepts explicatifs. Le premier est l'existence de structures d'opportunités politiques préalables qui ont balisé le chemin vers une intensification des réformes. Le second est l'existence d'un réseau d'événements, non pas liés mais mutuellement influençables, l'évolution de la situation dans un pays influençant significativement le cours des choses dans les autres pays.

Commençons par la première question. Pourquoi les peuples se révoltentils ? Sur ce point, Van Inwegen ${ }^{6}$ propose une distinction entre approches psychologiques et approches rationnelles. Du point de vue psychologique, Ted Gurr affirme que dans les sociétés caractérisées par des privations flagrantes ${ }^{7}$, quand les populations commencent à formuler des aspirations à une vie meilleure et qu'elles en perçoivent la possibilité, la frustration de ces aspirations les pousse à se rebeller contre les régimes en place. Du point de vue de l'école du choix rationnel, le défi qui se pose pour passer à l'action est celui du free rider - quand quelques-uns bénéficient de l'action des autres sans y avoir participé ${ }^{8}$. Le principal défi pour les tenants de cette approche est d'expliquer la participation des acteurs à ces actions collectives malgré l'existence d'un tel handicap. Pour résoudre ce défi, ils ont recours à plusieurs modèles - qu'ils présentent en général en termes de jeux - et qu'ils considèrent comme étant représentatifs de la réalité. Le problème est qu'aucune de ces approches ne prend en compte les comportements des acteurs dominants qui ont tout intérêt à faire avancer les choses, quitte à payer plus cher, puisqu'ils s'attendent à des gains plus conséquents. Les analyses de l'école du choix rationnel sont également statiques dans le temps et ne prennent pas en compte les relations sociales qui s'établissent entre les acteurs et leurs influences réciproques qui affectent les choix opérés tout le long du processus d'interaction.

C'est d'ailleurs ainsi que Charles Tilly, dans un article de 1992 analysant les mobilisations qui avaient conduit à la chute des régimes des pays de l'Est européen en 1989-1990, présente l'évolution des analyses portant sur les mobilisations 9. Il indique qu'elles ont d'abord adopté une approche historique de l'évolution des formes de l'action collective et de la conceptualisation des acteurs en tant que réseaux sociaux socialement constitués, évoluant avec le temps et les contingences politiques et idéologiques. La seconde évolution a

6. Inwegen P. V., Understanding Revolution, Boulder, Lynne Rienner, 2011.

7. Ibid., pp. 128-129.

8. Ibid., pp. 130-131.

9. Tilly C., "Réclamer Viva Voce », Cultures E Conflits, 5, printemps 1992, pp. 109-126. 
pris forme au travers des controverses entre ceux qui défendaient « les analyses complexes de l'action rationnelle » et ceux qui soutenaient des perspectives plus historiques, et qui cherchaient à comprendre les mobilisations selon leurs spécificités politiques, économiques et idéologiques. Un consensus a fini par se former entre ces deux composantes autour de l'idée qu'il est nécessaire de lier les processus historiques, économiques, politiques, idéologiques, et ceux de l'action collective. Tilly a également recours au concept de cycles de contestation pour affirmer que

[1]es revendications réussies ont tendance [...] à susciter d'autres revendications de la part d'acteurs différents, certains d'entre eux apercevant une ouverture jusqu'ici invisible ou inaccessible et d'autres au contraire ressentant une menace sur des positions acquises. L'expansion se poursuit jusqu'au "moment » (phase) où les concurrents se fixent, s'établissent, s'essoufflent, s'entretuent ou succombent à la répression étatique diligentée par ceux qui se sentent menacés par la complaisance des autorités [...] Le schéma de Tarrow se base sur une conception de la structure des opportunités politiques (political opportunity structure) contraignante pour tous les acteurs, variant d'un pays à l'autre et d'une année sur l'autre ${ }^{10}$.

Ainsi, les révolutions ne font qu'exacerber la fragilité des régimes en place et conduisent certains des bénéficiaires du statu quo à chercher des alternatives susceptibles de préserver leurs intérêts sur le long terme. Cette fragilité peut également s'expliquer, comme le propose Van Inwegen suivant Gurr, par des améliorations concrètes dans la vie des populations, améliorations dont les aspirations frustrées révèlent les limites.

Quand à la question de savoir pourquoi les mobilisations ont lieu à des moments précis, en dépit de l'existence préalable des conditions qui y mènent, le concept de structures d'opportunités politiques (qui lui aussi renvoie d'une certaine façon à l'argument de Van Inwegen et Ted Gurr) suggère que les choix des acteurs procèdent d'un contexte politique précis. Selon David Meyer ${ }^{11}$, ce contexte a tendance à favoriser certaines revendications et à en décourager d'autres. En d'autres termes, les choix des agents politiques sont limités et déterminés par un contexte politique précis, ainsi que des règles du jeu structurelles ${ }^{12}$. Meyer précise à ce propos que si certaines théories persistent à considérer que l'existence d'opportunités croissantes mais frustrées constitue le préalable nécessaire à toute mobilisation, d'autres estiment que le rôle de préalable à la mobilisation extra-institutionnelle est imprégné de l'existence d'une structure contraignante et limitée d'opportunités institution-

10. Ibid., p. 5.

11. Meyer D. S., "Protest and Political Opportunity", Annual Review of Sociology, 30, 2004, pp. 128-134.

12. Tilly C., op.cit., p. 128. 
nelles ${ }^{13}$. Il ajoute que l'absence de consensus sur les facteurs qui doivent être considérés comme partie prenante des opportunités politiques explique en partie ces divergences théoriques. Il indique ainsi que la consolidation du concept d'opportunité politique passe par la spécification tant des facteurs structurels établis en termes «d'opportunités » que des facteurs centrés sur le rôle des acteurs et qui opèrent en termes de «menaces », afin de pouvoir comprendre les dynamiques qui mènent aux révoltes populaires.

Le travail de Timothy Wickham-Crowley ${ }^{14}$ apporte un complément significatif à cette réflexion. Wikham-Crowley a cherché à expliquer pourquoi la révolution cubaine de 1959 ainsi que la révolution sandiniste de 1979 n'avaient finalement pas abouti à des changements de régimes dans d'autres pays de l'hémisphère occidental, en dépit de l'élan de révolte qu'elles avaient initié en Amérique Latine. En s'appuyant sur une analyse sociologique des jeux internes à chaque pays, il a souligné la nécessité d'analyser les interactions entre agents sociaux et l'imbrication de leurs actions afin d'expliquer pourquoi certains mouvements de la paysannerie latino-américaine se sont transformés en mouvements de guérilla, et pourquoi parmi ceux-là, deux seulement ont effectivement réussit à renverser les régimes en place. Selon lui, ce n'est pas parce qu'il y a injustice qu'il y a révolte, et ce n'est pas parce qu'il y a révolte qu'il y a chute de régime. Wickham-Crowley affirme ainsi que c'est la nature répressive du régime qui est au principe de la formation d'amples coalitions entre les groupes d'opposants. Il semble ainsi proposer une analyse plus centrée sur le rôle de "l'acteur », du moins dans le cas latino-américain. Pour lui, c'est l'interaction entre les menaces et la répression exercées par les régimes en place d'un côté, et les réactions des populations de l'autre, qui explique la mobilisation et la révolte des secondes contre les premiers.

La réflexion d'Antonio Gramsci est un troisième point d'entrée. Dans ses Lettres de prison ${ }^{15}$, il a cherché à expliquer les différentes modalités révolutionnaires, et en particulier le fait que la confrontation avec des structures politiques et des régimes différents requiert des mouvements révolutionnaires distincts, y compris pour arriver éventuellement à faire changer les régimes en place. Il opère ainsi une distinction entre guerre de position et guerre de mouvement. Pour faire face aux régimes où la bourgeoisie nationale est faiblement enracinée dans la société civile et dans lesquels la révolution peut atteindre ses objectifs en prenant l'État d'assaut, une guerre de mouvement est suffisante non seulement pour mettre à mal le régime, mais aussi le renverser. Quand le régime prend appui sur une structure hégémonique plus profondément enracinée du point de vue politique et culturellement acceptée par les masses, en

13. Ibid., p. 133-134.

14. Wickham-Crowley T. P., Guerrillas E Revolution in Latin America - A Comparative Study of Insurgents and Regimes Since 1956, Princeton, Princeton University Press, 1992.

15. Gramsci A., Selections from the Prison Notebooks, New York, International Publishers, 1992, 11 édition. 
revanche, le combat culturel contre les intellectuels de la classe dirigeante tentaculaire ne peut se faire par à-coups. Il ne sert à rien, selon Gramsci, d'essayer de faire chuter le régime par une guerre de mouvement. Dans ce cas précis, il est nécessaire d'avoir recours à des stratégies de longue haleine, de créer un bloc historique contre-hégémonique qui soit à même d'en découdre avec la légitimité du régime afin de la mettre en jeu et de permettre éventuellement au mouvement contre-hégémonique de faire face au groupe hégémonique et même le renverser. Le cas échéant, les tentatives de renversement par confrontations et rébellions directes seront toutes vouées à l'échec. Sans aucunement nier l'importance des facteurs structurels, Gramsci soutient que les acteurs politiques, qu'ils soient du régime ou de l'opposition, jouent un rôle crucial dans le choix des voies suivies ainsi que dans leur succès ou leur échec. Tilly semble d'ailleurs s'engager sur cette voie interactionniste lorsqu'il écrit :

La communication et les croyances partagées jouent un rôle critique au cours de ces processus révolutionnaires et quasi-révolutionnaires. Trois éléments sont amenés à se transformer au cours de ces processus : premièrement, la capacité de l'État ; deuxièmement, les croyances partagées parmi les acteurs politiques quant aux conséquences probables des diverses revendications et actions y compris les croyances au sujet de la capacité de l'État ; troisièmement, les capacités relatives des divers acteurs d'agir collectivement, qui comprennent d'une part la formation de nouveaux acteurs par création, mobilisation, ou coalition, et d'autre part, la diffusion des doctrines des connaissances techniques, et des militants ${ }^{16}$.

Le cas des révoltes arabes devient donc significatif à cet égard, puisqu'il appelle immédiatement la question suivante : avons-nous été en présence de structures d'opportunités politiques croissantes, d'ouvertures frustrées, expliquant que les peuples aient été amenés à se mobiliser et se révolter en 2011 ? Dans un contexte marqué par un grand déficit démocratique, pour emprunter l'expression utilisée par Marsha Posusney ${ }^{17}$, au sein duquel peu de réformes d'ouverture démocratique avaient été entreprises à l'exception de quelques changements cosmétiques réalisés sous la pression internationale, il est permis d'en douter. Selon Posusney, peu de nouveaux espaces politiques s'étaient ouverts aux populations dans le monde arabe pour leur permettre de formuler leurs frustrations. De plus, chacune des rares ouvertures observées a été suivie de reculs considérables.

Pour traiter de la seconde question, à savoir : pourquoi plusieurs pays arabes se sont embrasés après l'amorce, et surtout le succès de la révolution

16. Tilly C., op.cit., p. 7

17. Posusney M. P., “The Middle East's Democracy Deficit in Comparative Perspective”, in Posusney M. P., Angrist M. P. (eds.), Authoritarianism in the Middle East-Regimes and Resistance, Boulder, Lynne Rienner, 2005, pp. 1-18. 
tunisienne, il est intéressant de se tourner vers le travail de Theda Skocpol. Skocpol affirmait déjà en 1979 que des causes similaires ne mènent nécessairement pas aux mêmes conséquences ${ }^{18}$. Pour qu'une révolution ait lieu et qu'elle parvienne à renverser le régime en place, la présence d'un mouvement révolutionnaire, pour motivé et organisé qu'il soit, n'est pas suffisante. Les caractéristiques structurelles des régimes en place, et particulièrement les circonstances internationales, contribuent significativement à la réussite ou à l'échec des mouvements révolutionnaires. Pour Skocpol, ce sont les pressions externes qui finissent par fragiliser les régimes de l'intérieur, et les empêchent par la suite de réagir efficacement aux révoltes populaires. Il faut donc analyser les différents cas, pour ce qui concerne aussi bien la dimension interne des mouvements de mobilisation que celle des régimes, et à la lumière de la dimension internationale, pour arriver aux conditions historiques qui permettent d'aboutir ou non au renversement des régimes en place. Dans le cas qui nous préoccupe, la chute de Ben Ali en Tunisie, suivie de celle de Moubarak en Égypte, représente cette dimension externe cruciale qui va initier de nouvelles dynamiques dans les autres pays arabes, sans pour autant que les résultats soient similaires.

Ainsi, Tilly affirmait à propos des révoltes qui ont secoué les pays de l'Europe de l'Est en 1989-1990 que

[1]es sources de simultanéité entre mouvements sociaux qui se forment dans des pays différents résident tout d'abord dans des processus subis simultanément par les pays [...] puis dans les effets des luttes politiques sur les parties tierces, surtout en ce qui concerne les États [...] enfin dans les emprunts directs, de modèles d'action collective d'informations sur les conséquences (domestiques et internationales) de l'action collective, de croyances à l'égard des buts de l'action, et d'un savoir ou d'une capacité d'expertise des acteurs de l'action collective. Tous ces liens dépendent étroitement de l'histoire des relations entre les pays en question ${ }^{19}$.

Cette analyse coïncide significativement avec ce qui s'est passé dans le monde arabe en 2011. Ce sont les emprunts et les influences mutuels, aussi bien de la part des régimes que de la part des populations, qui ont créé une dynamique à première vue générale et homogène, mais néanmoins spécifique à chacun de ces pays.

Les questions auxquelles il faut donc répondre sont : y a-t-il eu des crises externes qui ont rendu certains régimes arabes plus vulnérables aux révoltes internes ? Y a-t-il eu des réformes préalables qui ont conduit à des opportuni-

18. Skocpol T., States and Social Revolutions, Cambridge, Cambridge University Press, 1979.

19. Tilly C., op.cit., p. 8. 
tés politiques jusqu'alors impensables, et des demandes plus substantielles de changement ont-elles été présentées ? La nature de certains régimes a-t-elle favorisé l'établissement d'une ample coalition contre eux ? La nature de certains régimes a-t-elle favorisé leur chute facile et immédiate?

Afin de pouvoir analyser ces questions et y répondre, il est important de prendre en compte plusieurs dynamiques, et plusieurs dimensions toutes liées entre elles : l'État/le régime en place, ses forces et ses faiblesses, les mouvements qui lui font face, et la dimension internationale. Ce sont les relations politiques et sociales qui s'établissent au sein de ces dynamiques et de ces dimensions qui expliquent le succès du changement en Tunisie, son succès tout à fait relatif en Égypte, le tournant violent dans des pays comme la Libye, la Syrie et le Yémen, et l'immobilisme apparent de la Jordanie et du Maroc, par exemple.

\section{Mobilisations et contre-mobilisations dans le monde arabe}

Les révoltes dans plusieurs pays arabes depuis la mi-décembre 2010 et l'immolation d'un jeune chômeur tunisien, ont déjà eu entre autres conséquences la chute de deux présidents, le Tunisien Zine Abidin Ben Ali et l'Égyptien Hosni Moubarak, la mise en place d'un nouveau pouvoir en Libye suite à la fuite et à l'assassinat du leader libyen Mouamar Kadhafi, la démission du président yémenite Ali Abdallah Saleh et sa substitution temporaire par son vice-président, en attente de nouvelles élections, la mise en difficulté du régime du président syrien Bachar al Assad ainsi que des mouvements de rue significatifs à Bahreïn, en Jordanie, au Maroc et à Oman. Enfin, des manifestations de type et d'intensité divers ont eu lieu en Algérie, en Arabie Saoudite, en Irak et dans les Territoires occupés de Cisjordanie et de Gaza. Ces mouvements inédits et suivis dans plusieurs pays du monde arabe ont poussé une partie de la presse internationale à créer sur leurs portails Internet des dossiers permanents traitant de ces différentes situations sous des intitulés tels que le «printemps de la révolution arabe » (Al Jazeera), la « région en révolte » (New York Times), la «vague de changement dans le monde arabe » (El País) ou bien encore l' «agitation dans le monde arabe »(CNN). Tous ces médias convoquent l'image d'un phénomène généralisé qui s'étendrait comme un incendie partout dans le monde arabe, et font référence aux idées de contagion, comme un virus, ou de prolifération, comme un cancer.

Force est de reconnaître que la tentation de l'image du cancer est grande : plusieurs pays arabes souffrent en apparence des mêmes maux (régimes autoritaires, peu ou pas de libertés individuelles, très haut niveau de corruption, jeunesse désabusée et au chômage, mais connectée au monde à travers les chaînes de télévision satellitaires et Internet), ce qui peut constituer une source d'inspiration réciproque, un appel à imiter les jeunes en Tunisie et en Égypte dans leurs révoltes contre leurs régimes respectifs. 
Utiliser le langage de la contagion ou de la prolifération des révoltes dans le monde arabe renvoie néanmoins à un automatisme qui ignore plusieurs aspects importants des relations politiques et sociales spécifiques à chaque contexte et à chaque réalité nationale. Ces phénomènes sont comparables dans la mesure où il s'agit de mouvements populaires, de formation relativement récente, qui sont peu ou pas organisés - du moins du point de vue structurel et institutionnel -, dans une région à dominante ethnique arabe. Mais il s'agit aussi de phénomènes très distincts les uns des autres. Ne pas apporter cette précision, c'est reléguer les différents acteurs de ces processus, leurs choix et leurs options au second plan, et accorder la prépondérance à la structure. Tilly, dans l'article précité, disait en référence aux événements en Europe de l'Est en 1989 qu'ils puisaient « dans un stock commun de connaissances et de références » et parlait « d'un réseau d'événements qui dépendent étroitement les uns des autres », ce qui décrit bien les mobilisations dans le monde arabe en 2011. Le type de mobilisations ainsi que leur séquençage, tout autant que leur persévérance, en Égypte d'abord, puis en Libye, en Syrie et ailleurs, ne peuvent être expliqués que par la réussite de la révolte populaire tunisienne, ainsi que par les conditions similaires dont souffraient ces différents peuples.

En quoi ces conditions sont-elles similaires ? Peut-on les renvoyer, dans des termes généraux, à une "condition arabe » ? Bien des analystes présupposent l'existence d'un monde arabe. Cette idée doit beaucoup au président Gamal Abdel Nasser d'Égypte ${ }^{20}$, mais ne reflète guère la grande diversité qui existe entre ces pays. Elle ne rend pas compte de la présence d'importantes minorités ethniques (Berbères, Kurdes) et religieuses (chrétiens, juifs, sans parler des minorités chiites), observation qui met en échec l'image d'une homogénéité ethnique, religieuse et historique au sein de la région. Mais ces pays partagent d'autres caractéristiques : ils sont tous ou presque dominés par des régimes non démocratiques, avec peu ou pas de libertés individuelles et d'expression, de hauts indices de corruption et une population très jeune qui considère ses perspectives d'avenir comme limitées. C'est donc un vécu commun que les jeunesses de ces pays peuvent revendiquer, vécu commun promu et confirmé par des véhicules satellitaires de télévision. Al Jazeera est l'exemple le plus connu de ces véhicules, mais il en existe d'autres, tel Al Arabia, une autre chaîne d'information, ainsi que des chaînes coraniques (tel Iqra), musicales (la plus fameuse étant Rotana), de variétés (MBC) et de films (ART entre autres), toutes suivies de très près dans plusieurs pays arabes ${ }^{21}$.

20. Muñoz G. M., op.cit., pp. 101-103.

21. Notons ici qu'Al Jazeera est aussi connue dans le monde arabe pour son journalisme que pour ses retransmissions de matchs de football, ayant acquis le monopole dans tout le monde arabe pour transmettre la coupe du monde de football ainsi que plusieurs compétitions européennes de prestige. Notons enfin que la chaîne Al Arabia avait été durement critiquée pendant les premiers soulèvements, surtout celui d’Égypte, pour son appui à peine voilé aux régimes qui ont fini par être déchus. Elle s'est largement rattrapée avec les révoltes en Syrie et en Libye. 
Malgré ces similarités, les événements de 2011 ont eu des effets différents sur ces pays. Alors que la révolte tunisienne naissante inspirait les autres peuples arabes (ou à dominante arabe), les régimes en place ont commencé à prendre des précautions. En Algérie, au Maroc, en Jordanie, en Syrie ainsi qu'en Égypte, une combinaison plus ou moins significative de mesures a été adoptée afin de satisfaire les demandes pressantes de plusieurs catégories populaires, et ce, avant même la chute de Ben Ali : certains salaires ont été augmentés, on a promis aux chômeurs des emplois, ou le cas échéant des indemnités de chômage, les prix des denrées de premières nécessité ont été bloqués et des remaniements ministériels ont eu lieu, y compris au plus haut niveau. Certaines de ces mesures ont été adoptées de manière préventive, avant que la rue ne commence à bouger. Les régimes et les peuples ont ainsi tiré des leçons différentes des mêmes événements. Certains ont conclu qu'il fallait faire des concessions importantes avant que celles-ci ne soient considérées comme insuffisantes et tardives, par contraste avec la situation en Tunisie et en Égypte, quand leurs présidents respectifs avaient promis de ne pas se présenter aux élections suivantes, concession qui aurait été significative en temps normal, mais qui fût prise trop tard pour avoir un quelconque effet positif sur les foules révoltées. D'autres régimes ont plutôt considéré que toute concession serait fatale, et que pour se maintenir au pouvoir, il fallait d'abord éviter de faire des concessions et réprimer aussi durement que possible les premiers soulèvements. Ainsi, alors que le président yéménite a évité, du moins dans un premier temps, de faire usage de la force à l'encontre des manifestants, et qu'au Maroc, le roi a rapidement annoncé un processus de réforme constitutionnelle pour avancer vers ce qu'il appelait la démocratie, les régimes libyen et syrien ont opté pour la manière forte et violente dès les premières manifestations.

Les peuples ont aussi réagi de manière différente : le fait que les Syriens, qui vivent sous le joug d'un régime répressif et très restrictifs du point de vue des libertés depuis la fin des années soixante, se soient rebellés après les Libyens, qui vivent sous le joug d'un régime tout aussi répressif et liberticide mais qui jouissent de conditions de vies autrement plus confortables grâce à la manne pétrolière en dit long sur la nécessité d'étudier soigneusement les conditions propres à chaque pays pour comprendre comment, pourquoi et quand les populations s'expriment. Le contraste des leçons apprises ne pouvait être plus grand entre Syriens et Libyens, puisqu'en Libye, les rebelles ont très vite eu recours aux armes, transformant la mobilisation initiale en rébellion armée contre le régime de Kadhafi, alors qu'en Syrie, malgré la répression violente 22 et largement condamnée par les acteurs internationaux ${ }^{23}$, les mobi-

22. Lire à ce propos les rapports «By All Means Necessary », «We Live as in War » «We've Never Seen Such Horror » et « Syria - Stop Torture of Children » de Human Rights Watch, disponibles sur le site internet de l'organisation (www.hrw.org/middle-eastn-africa/syria), ainsi que plusieurs rapports disponibles sur le site internet d'Amnesty International (www.amnesty.org/en/region/syria).

23. Lire les nombreuses déclarations et communiqués du Secretaire général des Nations unies à propos de la Syrie sur le site internet du Secrétaire Général (http://www.un.org/sg/). 
lisations hésitent à user de la violence et à avoir recours aux armes, à de rares exceptions près. Il faut aussi noter ici que si les Tunisiens ont rapidement commencé à scander "Ben Ali dégage », les Égyptiens à crier « Moubarak dégage » et les Syriens à exiger "soukout Annidam » (la chute du régime en arabe), les Marocains n'ont eu de cesse d'insister sur la nécessité de réformer le régime et de préserver la monarchie, d'où le soutien à un « roi qui règne mais ne gouverne pas ${ }^{24}$ ».

Une dynamique a donc été lancée en Tunisie et, à cause des similarités entre plusieurs pays de la région, régimes et peuples ont commencé à agir et réagir. Des leçons ont été apprises, aussi bien par les régimes que par les peuples, et leur application a engendré de nouvelles possibilités pour chacun de ces régimes et de ces peuples. Il ne s'agit donc pas d'une observation passive et unilatérale, mais d'une observation mêlée à l'action simultanée, aussi bien de la part des régimes que de la part des peuples. Une composante essentielle de ce vécu commun des peuples des pays dits arabes était le manque de crédibilité de la politique et des politiciens, question examinée dans la section suivante.

\section{Manque de crédibilité de la politique et des politiciens dans le monde arabe}

La dépolitisation avancée et voulue par les régimes en place constitue une importante similarité entre ces différents pays. La démoralisation de la politique et de la classe politique, la réduction, voire l'inexistence de débat politique constructif et serein tout autant que du droit à la différence et à la dissidence, la valorisation du consensus, ont abouti à un manque total d'encadrement politique, à l'abandon du champ politique aux opportunistes en tous genres, y compris, et à bien des égards, à ceux qui mobilisent la religion à des fins politiques ${ }^{25}$. La jeunesse actuelle ne sait rien ou très peu des luttes des générations précédentes, et surtout apprend peu des échecs continus de leurs prédécesseurs : elle a tendance à croire qu'elle est la première à oser affronter les régimes en place.

Selon Vickie Langohr ${ }^{26}$, les pays arabes ont connu ces dernières années une montée en puissance de la société civile comme principale opposition aux régimes, au vu de ce qu'elle appelle la complète marginalisation des partis politiques. Selon elle, les organisations de défense des droits humains, les organi-

24. Il faut toutefois noter quelques rares exceptions de manifestants qui ont demandé la chute du régime, exceptions rapidement réduites au silence par les propres organisateurs des manifestations. Ceux-ci ont d'ailleurs réservé leurs cris de « dégage » à certaines figures de l'entourage royal.

25. Bien des fois, la société civile est manipulée et instrumentalisée par le pouvoir afin de lui permettre d'atteindre ses objectifs de maintien du statu quo. Voir Pratt N., Democracy $\varepsilon$ Authoritarianism in the Arab World, Boulder, Lynne Rienner, 2007.

26. Langhor V., "Too Much Civil Society, Too Little Politics: Egypt and Liberalizing Arab Regimes”, Comparative Politics, 36-2, 2004, pp. 181-182. 
sations féministes ainsi que les groupes de protection de l'environnement ont pris la place laissée vacante par les partis politiques, et ont joué le rôle de principaux canaux de transmission entre gouvernés et gouvernants. Laila Alhamad 27 se démarque légèrement de Langohr quand elle remarque que le manque de perméabilité des voies politiques a conduit dans les sociétés arabes à l'exploration d'autres canaux afin de faire pression sur l'État, lui transmettre des demandes, sans pour autant faire ostensiblement de la politique, et ces canaux sont ceux de la société civile. Alhamad insiste donc ainsi sur la dépolitisation des sociétés arabes qui oblige la société civile à faire de la politique sans en avoir l'air, et ne voit pas nécessairement un remplacement des partis politiques par la société civile. Holger Albrecht semble confirmer l'idée d'Alhamad quand il précise que dans les pays du Moyen-Orient et d'Afrique $\mathrm{du}$ Nord, les sphères politique, économique et sociale sont souvent entremêlées. Selon lui, même quand les demandes sont de nature économique ou sociale, elles finissent par acquérir une dimension politique lorsqu'elles visent à influencer l'État et ses décisions ${ }^{28}$.

Du point de vue strictement politique, les régimes arabes ont ainsi mis en place des dispositifs à même de décrédibiliser la classe politique : des élections qui ne valent pas grand chose et des périodes électorales engendrant la mobilisation opportuniste de la classe politique qui cherche à se faire élire, mobilisation qui disparaît ensuite sans considération pour les électeurs qui ont participé au processus. Le résultat de cette configuration est la formation et la perpétuation d'une classe politique qui s'accommode du jeu politique proposé par les régimes en place, sans les contester, et sans contribuer à former une opposition réelle. La compétition politique est ainsi devenue un moyen au service de causes personnelles, comme en témoigne le cas de plusieurs clans familiaux qui se sont installés au pouvoir dans les pays arabes ${ }^{29}$. L'autre conséquence, tout aussi importante, est l'absence totale de crédibilité de cette classe politique auprès de la population et le manque de prestige de l'activité politique, comme l'analyse d'Abdeslam Maghraoui à propos du Maroc 30.

Il est vrai que dans ce monde arabe, ceux qui ont osé s'opposer aux régimes en place ont bien souvent été contraints à l'exil (Rachid Ghannouchi, le leader islamiste du parti Ennabda en Tunisie, ainsi que les membres de l'opposition libyenne et syrienne), jetés en prison (les leaders du Front islamique du salut (FIS) en Algérie), bannis (Abassi Madani et Ali Belhadj, et en Égypte,

27. Alhamad L., "Formal and Informal Venues of Engagement”, in Lust-Okar E., Zerhouni S. (eds.), Political Participation in the Middle East, Boulder, Lynne Rienner, 2008.

28. Albrecht H., “The Nature of Political Participation”, in Lust-Okar, E., Zerhouni, S. (eds.) Political Participation in the Middle East, Boulder, Lynne Rienner, 2008.

29. Les exemples les plus frappants étant ceux des familles Trabelsi en Tunisie (i.e., la famille de l'épouse de l'ex-président Ben Ali), la famille du président Syrien Béchar el Assad, ainsi que la famille du Premier ministre marocain, Abbas al Fassi.

30. Maghraoui A., "Depoliticization in Morocco", in Diamond L., Brumberg D. (eds.), Islam and Democracy in the Middle East, Baltimore, Johns Hopkins, 2003, pp. 67-75. 
Ayman Nour, qui « osa » se porter candidat contre le président Moubarak aux élections présidentielles de 2005). L'exil, l'emprisonnement ou le bannissement cohabitent également avec d'autres modalités, telles que la mise en place de cooptations plus ou moins directes de l'opposition par les régimes en place. Cette pratique est illustrée par le cas récent de la Jordanie, où le roi Abdallah a nommé en février 2011 un gouvernement avec quelques figures de l'opposition (bien que les islamistes aient, eux, refusé d'en faire partie), ou encore au Maroc, quand Driss Lachgar (un leader de l'Union socialiste des forces populaires qui défendait l'urgence d'une réforme constitutionnelle et voulait ouvrir des négociations entre les socialistes et leurs ennemis jurés, les islamistes, en vue des prochaines élections législatives) a été nommé ministre chargé des relations avec le parlement. Les moins connus ou les moins populaires ont, eux, été muselés et marginalisés au moyen d'arrestations abusives et de procès expéditifs. Le résultat de ces différentes stratégies est l'existence, dans la quasitotalité du monde arabe, d'une opposition «domestiquée », dans le sens où elle se soumet aux lignes rouges imposées par le régime et qu'elle s'accommode des espaces restreints qui lui sont autorisés ${ }^{31}$. Dans plusieurs de ces pays, la nomination illusoire de Premiers ministres issus d'élections permet aux régimes de faire jouer à ces derniers la fonction de fusible. Ainsi, le roi Abdallah de Jordanie, les présidents Moubarak en Égypte et El Assad en Syrie ont changé de Premier ministre pour essayer de calmer la rue (le premier a eu plus de succès, du moins temporaire, que les deux autres), alors que les manifestants marocains ont pendant des mois demandé, et même exigé, la chute du Premier ministre et de son gouvernement. La démoralisation de l'exercice politique a contribué à décrédibiliser la politique et des politiciens. Ainsi, quand on demande aux jeunes marocains s'ils militent dans des partis politiques, ou bien encore s'ils s'intéressent à la politique, ils répondent dans leur écrasante majorité par la négative. Par contre, ces mêmes jeunes savent tout de la politique française et assistent aux débats politiques et aux émissions politiques sur les chaînes de télévision françaises, ce qui traduit leur intérêt pour la chose politique, mais pas pour la politique marocaine. La conséquence de cette situation est perceptible dans le taux de participation catastrophique aux dernières élections législatives en date au Maroc. Celles de 2007 n'ont ainsi pas dépassé les $37,4 \%$, taux qu'on retrouve dans plusieurs autres pays arabes (35\% de participation aux législatives algériennes de 2007 et $27,4 \%$ aux législatives de novembre 2010 en Égypte). Il est d'ailleurs instructif de lire Samer Shehata et Saloua Zerhouni à propos des jeux politiques et électoraux, respectivement en Égypte et au Maroc ${ }^{32}$.

31. Les rapports successifs de Human Rights Watch sur les abus contre les droits de l'Homme au Maroc à la suite des attentas terroristes du 16 mai 2003 présentent un éloquent exemple de ce genre de musèlement politique à l'égard de l'opposition.

32. Shehata S., "Inside an Egyptian Parliamentary Campaign”, in Lust-Okar E., Zerhouni S. (eds.), Political Participation in the Middle East, Boulder, Lynne Rienner, 2008, pp. 95-120, et Zerhouni S., “The Moroccan Parliament”, in Lust-Okar E., Zerhouni S. (eds.), Political Participation in the Middle East, Boulder, Lynne Rienner, 2008, pp. 217-238. 
Les effets de cette démoralisation de la politique dans le monde arabe se sont révélés jusqu'au printemps 2011 dans une sorte de morosité doublée d'une forme de léthargie politique au sein des institutions légalement reconnues dans plusieurs de ces pays ${ }^{33}$. Non seulement les politiciens tendaient à accepter toutes les restrictions imposées par les régimes, mais leurs demandes étaient devenues de plus en plus modestes et symboliques. Ils ne croyaient pas dans la possibilité de changements radicaux et s'abstenaient de les appeler de leurs vœux. Ainsi, en Tunisie comme en Égypte, l'opposition légale et tolérée jusqu'alors par les régimes de Ben Ali et Moubarak n'a à aucun moment rejoint les manifestants dans les rues ${ }^{34}$. Quant au Maroc, aucun parti politique représenté au Parlement n'a officiellement soutenu les jeunes du 20 février. Quelques chefs de partis s'y sont même ouvertement opposés, et certains des rares politiciens à avoir rejoint les rangs des manifestants ont eu maille à partir avec leurs partis politiques ${ }^{35}$.

Le champ politique était donc libre pour des leaders alternatifs, bien souvent issus du champ religieux. En effet, les systèmes politiques étaient tellement verrouillés que pour exprimer une opposition significative aux régimes, il fallait se situer en dehors du système politique. Nicola Pratt identifie d'ailleurs à ce propos une transformation de la société civile : le mouvement étudiant, les partis politiques, les syndicats, la mouvance islamiste luttaient pour pouvoir s'exprimer plus librement par rapport aux régimes, mais sans grand succès. Selon Pratt, ce sont surtout les organisations de résistance culturelle (berbères au Maghreb, kurdes en Irak et en Syrie, chrétiennes ou chiites là où ces communautés existent), ainsi que les mouvements féministes et de défense des droits humains ${ }^{36}$ qui ont réussi à explorer des espaces publics d'action et sont devenus les principaux canaux de résistance aux régimes, au détriment des partis politiques ${ }^{37}$. Cependant, Pratt ajoute que face à cette transformation de la société civile s'est opérée une réorganisation de l'autoritarisme dans

33. Brownlee J., “The Decline of Pluralism in Mubarak's Egypt”, in Diamond L., Plattner M. F., et Brumberg D. (eds.), Islam and Democracy in the Middle East, Baltimore, Johns Hopkins, 2003, pp. 48-57, et Maghraoui A., "Depoliticization in Morocco", in Diamond L., Brumberg D. (eds.), Islam and Democracy in the Middle East, Baltimore, Johns Hopkins, 2003, pp. 6775.

34. Pour plus de détails, consulter le déroulement de ces divers développements dans The Guardian (http://www.guardian.co.uk/world/interactive/2011/mar/22/middle-east-protestinteractive-timeline) ainsi que sur le site de la chaîne de télévision américaine PBS réservé aux évènements du monde arabe de 2011 (http://www.pbs.org/newshour/timeline/uprising/).

35. Ce fut le cas de Mustapha Ramid, alors parlementaire de l'opposition islamiste du Parti de la justice et du développement (PJD), et qui est par la suite devenu ministre de la Justice au sein du nouveau gouvernement marocain, qui est entré en conflit direct et ouvert avec le secrétaire général de son parti. Il faut aussi noter que la jeunesse du parti socialiste ainsi que celle du PJD ont appuyé plusieurs revendications du Mouvement du 20 février. Les jeunesses de ces deux partis politiques se sont ainsi positionnées contre les aînés de leurs partis respectifs. Lire à ce propos Bekkali A., L'An 1 de la cyber démocratie au Maroc - 20 février 2011, Casablanca, Hammouch, 2012.

36. Notamment en Tunisie, en Algérie et en Égypte, où ces mouvements ont gagné en vigueur pendant les années 1990 avec la fondation de nouvelles organisations, indépendantes du pouvoir, particulièrement actives dans la défense des idéaux universels des droits humains.

37. Pratt N., op.cit. (voir en particulier le chapitre 5 où ces differents mouvements sont discutés). 
le monde arabe, non seulement à travers la répression et la réduction des espaces de liberté, mais aussi au travers des incitations à créer des organisations de la société civile «domestiquées » puisque soutenues et manipulées par les régimes, dont la fidélité leur est donc acquise. Ainsi, quand les régimes veulent montrer qu'ils sont à l'écoute de leurs peuples, ils convoquent tous les représentants de la société civile, noyant ainsi les revendications des groupes qui leur sont opposés dans les concerts de louanges chantées par leurs alliés et clients.

De plus, avec les déconvenues du nationalisme arabe (aussi bien le baasisme que le nassérisme), du communisme (suite à la chute de l'Union soviétique et la fin de la guerre froide) et des partis de gauche (ce qui est très évident au Maroc, où l'ex-opposition socialiste a fait partie des coalitions gouvernantes entre 1998 et 2011), dans plusieurs pays arabes, les seules alternatives crédibles furent de nature religieuse. C'est le cas en Tunisie avec Ennabda, en Égypte avec les Frères musulmans, en Algérie avec le FIS, et au Maroc avec le mouvement $A l A d l$ wal Ibsan de Cheikh Yassine, pour ne citer que les pays d'Afrique du Nord. Bien entendu, et comme évoqué précédemment, la classe politique actuelle contribue à bien des égards à cette démoralisation de la politique, en se permettant d'être cooptée et instrumentalisée, fréquemment pour des maroquins ministériels vides et sans pouvoir. Cet opportunisme de la classe politique ne joue ainsi pas en sa faveur, comme en atteste le manque d'intérêt qu'ont suscité Amr Moussa (rien moins que le précédent secrétaire général de la Ligue arabe et qui a tenté de se présenter en tant qu'opposant et victime du régime du président Moubarak lors de l'occupation de la place Tahrir) et Mohamed el Baradei (ex-directeur général de l'AIEA, Agence internationale de l'énergie atomique) en Égypte.

\section{À propos de pressions internationales et de l'islam politique}

Quel rôle ont joué l'Occident en général, les États-Unis et l'Europe occidentale en particulier, dans l'évolution politique de ces dernières années ? L'islam politique a-t-il eu un rôle dans ces différents évènements ?

L'Occident, tout d'abord, s'est accommodé pendant très longtemps des dictatures dans les pays arabes, aussi bien pour des raisons d'ordre culturel que géopolitique. Aux yeux de l'Occident, l'exception arabo-musulmane se justifiait par la culture simultanément tribale et patriarcale arabe, culture jugée totalement incompatible avec la démocratie occidentale, ainsi que par l'islam, qui est en même temps religion et État, pour se tenir à une traduction intégrale de l'expression «Din oua Daoula » souvent citée, qui évoque les dimensions simultanément spirituelles et politiques de la religion musulmane ${ }^{38}$. Ainsi, du

38. L'œuvre de Bernard Lewis en général, ainsi que celle de Samuel Huntington en relation au choc des civilisations, illustrent parfaitement cette perception occidentale : Lewis B., Islam 
point de vue culturel, une interprétation conservatrice du concept de "Assabya », déjà discutée au XIVe siècle par Ibn Khaldun, définissait la société arabe comme étant une société tribale, où les liens de sang sont la base de toutes les relations, et où la hiérarchie est basée sur le respect des plus vieux, et donc des plus sages. Ce sens aigu de l'importance de la communauté tribale était censé aller contre certaines valeurs de la démocratie occidentale. L'islam, dont certains évoquent les préceptes politiques et juridiques comme référence pour leurs actes politiques, empêcherait aussi le débat politique entre individus et groupes. Selon cet argument, si ce qui nous vient de Dieu est indiscutable, nous devons nous contenter d'interpréter correctement ses commandements. En fait, Edward Saïd suspectait déjà cet essentialisme envers l'Orient en général, et l'Arabe en particulier, de figer des réalités prétendument inébranlables pour être considérées comme profondément ancrées dans cet Orient ${ }^{39}$. Mais selon lui, et les événements de 2011 lui donnent d'une certaine façon raison, il n'y a rien d'essentiel dans cette identité arabe ; ces représentations ont plus à voir avec la façon dont l'Occident voit l'Orient. Par exemple, et dans la lignée de ce que disait déjà Saïd, il faut noter que ces interprétations de la culture tribale et de l'islam ne tiennent pas compte du fait que les chefs de tribu doivent garantir leur légitimité en défendant leurs tribus, et que leur pouvoir n'est ni absolu ni permanent. Elles considèrent aussi que ce concept tribal repose sur une homogénéité qui ne s'est jamais vérifiée, aussi bien par les mariages que par les autres moyens effectifs de miscégénation ${ }^{40}$. L'islam présenté comme épouvantail pour le changement politique s'est également révélé être un mythe, et l'évolution démocratique de l'Indonésie et de la Turquie montre bien la limite de cet argument de l'exception musulmane.

Du point de vue géopolitique, les régimes en place dans les pays arabes ont été perçus et représentés, aussi bien par l'Occident que par eux-mêmes, comme des remparts contre les extrémismes en tous genres, et en particulier contre l'extrémisme islamique. Le spectre des menaces du terrorisme et des États dits «parias », ceux-là mêmes qui sont accusés d'appuyer les terroristes et de collaborer avec eux, a ainsi été systématiquement brandi pour justifier l'appui à des régimes peu regardants sur les droits de l'Homme et la démocratie. Ainsi, quand le FIS est sorti vainqueur du premier tour des élections législatives en Algérie en décembre 1991, et qu'il paraissait en mesure de remporter une victoire significative au second tour en janvier 1992, le coup d'État militaire qui a fait avorté le processus électoral algérien a été bien accueilli par les pays occidentaux, et notamment par la France et les États-Unis, où se répétait l'adage selon lequel l'Occident appuyait le principe «d'une personne, une

and the West, Oxford, Oxford University Press, 1993, et Huntington S., The Clash of Civilizations and the Remaking of World Order, New York, Simon \& Schuster, 1996.

39. Said E., Orientalism, New York, Vintage, 1979, et en particulier l'introduction du livre où Said élabore explicitement cet argument.

40. Cherkaoui M., Morocco and the Sahara - Social Bonds and Geopolitical Issues, Oxford, Bardwell Press, 2008, 2e éd., pp. 157-160. 
voix », mais pas si ce principe ne devait s'appliquer qu'une seule fois. En somme, les islamistes étaient suspectés de vouloir conquérir le pouvoir par l'intermédiaire des urnes pour mieux supprimer les élections après leur victoire. En Algérie donc, l'Occident a préféré se bercer de l'illusion qu'un coup d'État militaire pouvait protéger la démocratie ${ }^{41}$. La démocratie n'était envisageable pour les Algériens que dans la mesure où ils élisaient des leaders acceptables aux yeux de l'Occident. Un autre exemple de ce genre a suivi les élections parlementaires dans les Territoires occupés de Cisjordanie et de Gaza, quand le mouvement Hamas a remporté les élections législatives en 2006 mais que son gouvernement, issu des urnes, avait été boycotté aussi bien par les États-Unis que par l'Union européenne et ses États membres, et sanctionné à bien des égards ${ }^{42}$. L'Occident n'a accepté comme éventuel partenaire en Palestine que le Fatah, à savoir ceux-là mêmes que les Palestiniens avaient rejetés dans les urnes. Encore une fois, la démocratie n'était bonne dans une partie du monde arabe que si elle permettait d'élire des leaders acceptables aux yeux de l'Occident.

Dans le même temps, les demandes de démocratisation, ou du moins de libéralisation du monde arabe n'ont cessé depuis la fin de la guerre froide, au moment où plusieurs pays, sur différents continents, évoluaient vers des régimes plus démocratiques. Après l'invasion de l'Irak par les États-Unis et leurs alliés en 2003, ces pressions pour plus de démocratisation se sont accrues sur le monde arabe. De ce point de vue, les incitations ou les pressions en faveur de la démocratie, qu'elles aient eu pour origine les États-Unis ou l'Europe, n'ont jamais cessé. En ce qui concerne l'Europe, le processus lancé à Barcelone en $1995^{43}$ et appelé « dialogue euro-méditerranéen » comptait au nombre de ses piliers l'amélioration des conditions politiques et des droits de l'Homme des pays du pourtour sud de la Méditerranée. Â cette époque déjà, l'Union européenne évoquait la nécessité pour ces pays d'aller vers plus de démocratie. Bien que l'imposition de ces normes par l'Europe à ses partenaires et voisins du Sud soit l'objet de bien des critiques, et notamment quant à leur caractère impérial ${ }^{44}$, tous les forums et débats, qu'ils soient publics ou académiques, et qui furent soutenus par l'Europe, tous les liens formels et informels entre les sociétés civiles des deux bords de la Méditerranée, ainsi que les pressions du Parlement européen aussi bien sur les autorités européennes que sur

41. Roberts H., The Battlefield Algeria, 1988-2002: Studies in a Broken Polity, Londres, Verso, 2003.

42. Musu C., “The EU Strategy for the Middle East Peace Process in the Post-9/11 Era”, in European Union Policy towards the Arab-Israeli Peace Process: The Quicksands of Politics, Basingstoke, Palgrave Macmillan, 2010 ; Tocci N., "What Went Wrong? The Impact of Western Policies towards Hamas \& Hizbollah”, CEPS Policy Brief, 135, juillet 2007, Bruxelles, (http://shop.ceps.eu/BookDetail.php?item_id=1523).

43. Fernández H. A., Youngs R., “The Barcelona Process: An Assessment of a Decade of the Euro-Mediterranean Partnership", Real Instituto Elcano, p. 5 (http://www.realinstitutoelcano.org/analisis/849/Amirah-Youngs849.pdf).

44. Zielonka J., Europe as Empire - The Nature of the Enlarged European Union, Oxford, Oxford University Press, 2006, pp. 57-59. 
les autorités des pays d'Afrique du Nord, ont sans doute contribué à valoriser la démocratie parmi les citoyens des pays d'Afrique du Nord.

Quant aux États-Unis, ils ont exercé une forte pression après leur invasion de l'Irak pour que des élections soient organisées dans les pays arabes. Ainsi, l'initiative du gouvernement Bush pour la démocratisation du monde arabe et la tenue d'élections - qui s'est notamment traduite par des pressions explicites sur des alliés aussi différents que l'Égypte, la Jordanie, l'Arabie Saoudite, et le Maroc - ont poussé ces pays à faire semblant de se démocratiser. Le régime égyptien a autorisé la participation du parti Wafd aux élections législatives de 2005, alors qu'en Arabie Saoudite, des élections municipales ont été organisées en 2005 45. En Jordanie (en 2007 et 2010), au Maroc (en 2007), mais aussi au Koweït (en 2006, 2008 et 2009), des élections législatives pluralistes et relativement transparentes ont été organisées, ce qui a valu à ces pays d'être cités par le président Bush en exemples aux autres pays de la région. Ces ouvertures libérales ont créé des structures d'opportunité politique dans la région dans la mesure où un champ de libertés accrues, même sous surveillance, s'est mis en place, permettant à de nouveaux acteurs de s'exprimer publiquement et à des initiatives jusqu'alors impossibles ou impensables de se développer. Ces ouvertures ont ainsi permis à ceux qui rejetaient le statu quo de communiquer entre eux et de s'organiser.

Depuis le début des événements de l'année 2011, les pressions internationales sur les régimes arabes visant à éviter la répression et encourager la voie des réformes politiques se sont accrues, bien que suivant des logiques différentes selon les pays. Ainsi, le discours de la secrétaire d'État américaine Hillary Clinton à Doha la veille même de la chute du président Ben Ali ou, de manière plus expressive encore, le discours du président Obama au département d'État le 19 mai 2011 (et qui est apparu comme une réaffirmation de son discours du Caire du 4 juin 2009), représentent autant de signes explicites, sur le plan du discours, du soutien des États-Unis à la démocratisation du monde arabe. Des pressions et des mises en garde - parfois publiques et explicites, comme dans les cas de la Libye et de la Syrie, parfois implicites et à travers des canaux diplomatiques, comme dans le cas du Maroc - ont été adressées par les États-Unis sur ces différents régimes pour les exhorter à ne pas réprimer violemment les manifestants. Simultanément, les ouvertures politiques des régimes - comme dans le cas de la Jordanie et du Maroc - sont aussi mises en valeur, du moins en public. Ces pressions peuvent-elles être considérées à l'origine des évènements actuels dans le monde arabe ? Leur effet ne doit sans doute pas être négligé. Mais leur faible impact et les contradictions qui leur

45. Il faut noter cependant que ces élections municipales en Arabie Saoudite furent organisées dans des conditions hautement surveillées et contrôlées par le pouvoir et ont servi à élire des pouvoirs municipaux avec des prérogatives très limités. Kraetzschmar H. J., "Electoral rules, voter mobilization and the Islamist landslide in the Saudi municipal elections of 2005", Contemporary Arab Affairs, 3-4, 2010, pp. 520-522. 
sont inhérentes - notamment quand il s'agit par exemple d'appuyer la reconnaissance de la Palestine comme État membre de l'Organisation des Nations unies (ONU) - sont mis en exergue dans les pays arabes, indiquant ainsi que leur portée est limitée, comme les faits l’ont indiqué sur le champ.

En outre, les hésitations occidentales par rapport aux évènements en Tunisie, en Égypte et même en Libye et en Syrie, ont mis en évidence l'importance des calculs et des intérêts en jeu. Ainsi, et sous prétexte de ne pas vouloir s'immiscer dans les affaires internes de la Tunisie, la France a montré très peu de fermeté à l'égard de la violente répression des manifestations par le régime de Ben Ali. Quant aux États-Unis, s'ils se sont montrés moins complaisants à l'égard du régime de Ben Ali que la France, ils ont par moments tergiversé face aux événements de la place Tahrir au Caire, et le gouvernement Obama a semblé initialement tenir à ce que ces événements ne conduisent pas à la chute du président Moubarak ${ }^{46}$. Quant à la Libye, la population de Benghazi s'est demandée pendant près d'un mois ce qu'attendaient l'Occident et l'ONU pour leur venir en aide et les protéger des attaques du colonel Kadhafi, puisque le Conseil de sécurité des Nations unies n'a permis à l'OTAN d'imposer une zone d'exclusion aérienne que près de cinq semaines après le début de la répression sanglante de la population libyenne. Même si les cas de la Libye et de la Syrie sont différents ${ }^{47}$, l'immobilité du même Conseil de sécurité face à la répression dans ce pays suscite le même genre d'interrogations à propos des tergiversations internationales en général, occidentales en particulier, vis-à-vis de la démocratisation du monde arabe. Ainsi, quand d'autres intérêts sont en jeu, comme par exemple un risque d'interpréter une intervention internationale comme visant exclusivement le contrôle direct du pétrole libyen ou bien encore, d'affaiblir la Syrie, et du coup, le Hamas, le Hezbollah et l'Iran, et en dernière instance, favoriser Israël, les massacres subis par les populations arabes passent au second plan ${ }^{48}$.

46. Il est vrai que plusieurs alliés d'Israël, surtout parmi les Républicains, ont vivement reproché au président Obama d'avoir abandonné un fidèle allié, et ont par la suite répété qu'Obama avait perdu l'Égypte, dans le même style que les mêmes Républicains avaient soutenu en 1979 que le président Carter avait perdu l'Iran, le Nicaragua et l'Afghanistan. Lire notamment Landler, M., “A Region s Unrest Scrambles US Foreign Policy”, The New York Times, 25 janvier 2011 (http://www.nytimes.com/2011/01/26/world/26diplo.html?_r=1), ainsi que Cooper, H. et Landler, M., "U.S. Trying to Balance Israel's Needs in the Face of Egyptian Reform”, The New York Times, 4 février 2001 (http://www.nytimes.com/ 2011/02/05/world/middleeast/05israel.html).

47. Il faut noter par exemple qu'à la suite d'une longue période de marginalisation et d'isolement dû notamment à des sanctions de l'ONU, le régime de Khadafi avait réussit à réintégrer la scène internationale, réussissant en particulier à établir d'importants nouveaux partenariats commerciaux et d'investissements avec plusieurs pays européens, ce qui avait valu au leader libyen maintenant déchu d'être reçu en grande pompe par de nombreux chefs d'états et de gouvernements occidentaux. La situation géopolitique de la Syrie accentue ces différences, puisque ce pays partage d'importantes frontières avec Israël, frontières très tendues d'ailleurs, en plus de jouir d'une influence importante sur des groupes comme le Hamas dans les territoires occupés de Cisjordanie et de Gaza, et le Hezbollah au Liban, ainsi que d'être l'une des rares mais importants alliés du régime iranien.

48. Il faut cependant noter ici que la lenteur de l'ONU à agir dans les questions libyennes et 
Quant à l'islam politique et à l'incompatibilité entre islam et démocratie, certains ont longtemps craint qu'une fois arrivés au pouvoir par des voies démocratiques, les mouvements et partis islamistes n'imposent leurs vues et positions, et nient - ou du moins limitent - les voies de la participation politique, et donc de la démocratie. Ce spectre, cet épouvantail, a longtemps été brandi, aussi bien par les régimes dans le monde arabe que par l'Occident, pour inhiber les demandes de démocratisation dans la région. Qu'en est-il ? Jusqu'au début de 2011, la répression était la réponse de presque tous les régimes du monde arabe aux mouvements islamistes, particulièrement en Tunisie et en Égypte. Les leaders du mouvement Ennahda en Tunisie et des Frères musulmans en Égypte étaient soit en exil, soit en prison dans leurs pays respectifs. Les seules voies d'action étaient donc nécessairement clandestines.

Les révoltes, aussi bien en Tunisie qu'en Égypte, ont néanmoins jeté une nouvelle lumière sur ces mouvements. En Tunisie, très peu de femmes voilées ont été vues parmi les manifestants. En Égypte, et peut-être parce que le ministère de l'Intérieur a tout de suite accusé les Frères musulmans d'être derrière les manifestants, l'organisation jusque-là illégale a mis du temps à rejoindre les rangs des manifestants présents sur la place Tahrir. Même après avoir pris part aux manifestations, les Frères musulmans égyptiens ont accepté, l'appel à la négociation du régime le 6 février et se sont réunis avec les représentants du président Moubarak, alors que d'autres opposants avaient refusé de participer à cette réunion et que les manifestants de la place Tahrir demeuraient très méfiants. Le fait d'avoir accepté cela montre d'ailleurs que les Frères musulmans faisaient de la politique, qu'ils étaient prêts à négocier et à faire des concessions, et qu'en somme, ils n'étaient pas les extrémistes qu'on voulait bien faire croire. Il faut aussi remarquer que nombre de slogans proférés par les manifestants place Tahrir n'étaient nullement religieux, peu sectaires et qu'ils faisaient preuve d'ouverture et de tolérance religieuse. Au Maroc aussi, les militants et les leaders du mouvement islamiste Al Adl wal Ibsan, non reconnu par l'État mais dont l'action est tolérée, ont tout d'abord hésité à rejoindre les rangs des manifestants du 20 février ${ }^{49}$. Ils ne se sont décidés que l'avant-veille des premières manifestations. En fait, en Tunisie, en Égypte comme au Maroc, les manifestations n'avaient aucune dimension confessionnelle. Les manifestants n'avaient rien contre l'islam, mais ne voulaient pas donner un caractère religieux à leurs messages et leurs slogans. Ceci ne veut certainement pas dire que les islamistes soient en perte de vitesse ou qu'ils n'auront aucun rôle dans les futurs systèmes politiques des pays arabes. Au Maroc, par exemple, quand la première mouture de la Constitution a été communiquée aux partis politiques, le Parti de la justice et du développement

syriennes est loin d'être due exclusivement à des tergiversations occidentales. Ainsi, les vetos russes et chinois au sein du Conseil de sécurité de l'ONU à toute action décisive des Nations unies en Syrie jouent aussi un rôle fondamental dans le manque de réaction effective de ce qui s'appelle en général de communauté internationale.

49. Bekkali A., op.cit. 
(PJD), parti à tendance islamiste autorisé légalement à participer au jeu politique marocain, s'est emparé du devant de la scène et a même menacé de voter contre la nouvelle Constitution si ce qu'ils appelaient « l'identité musulmane du Maroc » n'était pas clairement réitérée. Le PJD obtint gain de cause. D’ailleurs, lors des élections législatives en Tunisie, en Égypte, ainsi qu'au Maroc, les partis politiques à base religieuse ont réalisé de très bons scores et remporté ces élections - sans pour autant obtenir des majorités absolues, et donc sans pouvoir dominer leurs systèmes politiques respectifs, ou gouverner seuls. Les avancées politiques et électorales de partis islamistes modérés sont précisément dues à cette modération, tout autant qu'à leurs revendications qui ont ciblé la corruption, la transparence et la dignité, sans s'attaquer, notamment en Égypte, ni à l'Occident, ni aux États-Unis, et encore moins à Israël. Ces partis ont aussi maintenu leurs distances par rapport au salafisme, et surtout à sa composante jihadiste. Cependant, dans le nouveau contexte politique du monde arabe, il est nécessaire de réévaluer le rôle de la mouvance islamiste et de cesser tant de l'exagérer que de l'isoler.

\section{Et les événements de 2011 alors?}

Dès lors, comment analyser ce qui s'est passé dans ce monde arabe ? Comment expliquer les différences de réaction entre les pays arabes ? Comment expliquer que la Tunisie se soit débarrassée non seulement de Ben Ali, mais aussi de son régime, et que les Tunisiens aient réussi à imposer l'élection d'une assemblée constituante, alors que les Égyptiens sont seulement parvenus à faire chuter Moubarak (ce qui est loin d'être négligeable), tandis que les militaires se présentent toujours en garants de la transition vers un nouveau régime, avec tout ce que cette tutelle militaire représente en tant que passif de l'ancien régime, aussi bien en termes de corruption qu'en termes de manque de transparence institutionnelle et donc démocratique ? Comment analyser le contraste avec les autres pays ayant connu des révoltes - les Libyens qui furent obligés de prendre les armes, alors que les Syriens et les Yéménites confrontés à des répressions violentes et généralisées, ou encore les Marocains du Mouvement du 20 février pris de cours et actuellement dos au mur ?

Il faut insister sur l'effet d'entraînement des révolutions en Tunisie et en Égypte sur le reste de l'Afrique du Nord et du Moyen-Orient. Comme le disait Tilly à propos des pays d'Europe de l'Est, les expériences similaires vécues par les différents peuples de la région se sont retrouvées prises dans un réseau d'événements dépendant les uns des autres, échangeant les exemples aussi bien en termes de modèles que de modes d'action ou de leçons à apprendre, bien que ces leçons aient été de nature différente. Le fait que les Tunisiens aient pu déboulonner Ben Ali, leader d'un régime policier et répressif, a représenté une source d'inspiration pour les autres peuples d'Afrique du Nord. Le fait que les Égyptiens aient pris exemple sur les Tunisiens, qu'ils soient sortis dans les rues pour manifester et éventuellement demander la chute de 
Moubarak, qu'ils aient utilisé, tout comme les Tunisiens, les nouveaux réseaux sociaux sur Internet tel que Facebook et Twitter, forçant même le gouvernement Moubarak, dans un geste de désespoir, à suspendre pour quelques heures l'utilisation d'Internet ainsi que des téléphones portables, et qu'ils y soient arrivés, est venu renforcer le sentiment qu'après tout, ces régimes arabes n'étaient pas aussi inamovibles qu'on ne le pensait, et qu'ils ne résisteraient pas à une pression constante et puissante de la rue. Deux leçons différentes, voir contradictoires, ont néanmoins été tirées par les régimes arabes de la chute des présidents Ben Ali et Moubarak.

La première était que toute concession de la part des régimes est potentiellement fatale. En effet, quand on suit la ligne des événements en Tunisie et en Égypte, aucune concession, aussi significative qu'elle puisse être, ne fut en mesure de calmer les foules. Quand le président Ben Ali est intervenu pour la première fois à la télévision le 28 décembre, 11 jours après le début des manifestations, il a eu beau renvoyer trois ministres et trois gouverneurs, les manifestants n'ont pas reculé. Quand, le 13 janvier, il a promis de ne pas se présenter aux élections présidentielles de 2014, promesse que les Tunisiens auraient beaucoup appréciée quelques semaines auparavant, les manifestants ne se sont pas montrés satisfaits et ont demandé plus. La même chose s'est produite le 14 janvier, quand le président Ben Ali a démis le gouvernement de ses fonctions et annoncé des élections législatives dans les six mois suivants : au lieu de reculer, les manifestants ont demandé plus, et le soir même, ils ont obtenu ce qu'ils voulaient avec la fuite du président vers l'Arabie Saoudite 50.

En Égypte, un scénario identique s'est produit, avec des concessions du président qui auraient pu être considérées comme importantes, mais qui, dans le contexte précis des manifestations de rue, étaient perçues comme largement insuffisantes. Il faut dire que pendant toute la crise, le président Moubarak a semblé en net décalage avec le peuple égyptien. Ainsi, le 28 janvier, quatre jours après le début des manifestations, et alors que les manifestants attendaient des mesures concrètes (certains sur $\mathrm{Al}$ Jazeera parlaient même de l'imminente annonce de la démission de Moubarak), le président égyptien a prononcé un discours sans contenu, dont les principales mesures concernaient la protection de sa présidence plutôt que la satisfaction des demandes populaires. Un nouveau Premier ministre et un nouveau vice-président furent ainsi annoncés, tous deux des militaires fidèles à Moubarak. Et quand, le 1er février, il a annoncé, tout comme le président Ben Ali, qu'il n'allait pas se présenter aux élections présidentielles, la promesse a eu très peu d'effets sur les manifestants de la place Tahrir. Même la démission de son fils Gamal Moubarak du parti au pouvoir le 5 février n'a pas eu d'effets majeurs alors que

50. Pour plus de détails ici aussi, consulter le déroulement de ces divers développements dans The Guardian (http://www.guardian.co.uk/world/interactive/2011/mar/22/middle-east-protestinteractive-timeline) ainsi que sur le site de la chaîne de télévision américaine PBS dédié aux évènements du monde arabe de 2011 (http://www.pbs.or/newshour/timeline/uprising/). 
cela signifiait clairement son intention de ne pas se présenter aux élections présidentielles ${ }^{51}$. Le déphasage entre le président Moubarak et le reste du pays n'a jamais paru aussi évident que le 10 février, quand tous attendaient sa démission formelle, et qu'au lieu de cela, il a répété sa promesse que ni lui, ni son fils n'allaient se présenter aux élections présidentielles. Avant même la fin du discours, les télévisions ont montré la réaction immédiate des manifestants de la place Tahrir, qui ont commencé à huer, siffler et jeter leurs chaussures en l'air en signe de désespoir, mais aussi pour lui opposer une fin de non-recevoir.

La confiance entre les deux présidents et les peuples tunisien et égyptien, ou du moins ceux qui manifestaient dans les rues, avait été rompue et leurs paroles n'avaient plus de valeur aux yeux des manifestants. Certains régimes arabes ont donc appris à leur dépens que faire des concessions ouvrait la voie à une fin inéluctable, puisque les concessions des présidents Ben Ali et Moubarak les ont menés tous deux vers la porte de sortie. Le colonel Kadhafi en Libye, ainsi que les présidents Assad en Syrie et Salah au Yémen, ont ainsi décidé de ne faire aucune concession significative, et de contrer les manifestants par la force. À leurs yeux, seule une répression massive et immédiate était en mesure de sauver leurs régimes.

D’autres ont tiré une autre leçon, tout à fait opposée à la précédente, selon laquelle les concessions tant de Ben Ali que de Moubarak avaient été faites trop tardivement et étaient insignifiantes au vu de ce que demandaient les manifestants. Les réformes engagées ou promises par les deux présidents préfiguraient des structures d'opportunités politiques uniques et favorables à des changements bien plus profonds que les présidents déchus n'étaient en mesure de promettre. Ainsi, et selon cette autre logique, si réformes il y a, il faut qu'elles anticipent les événements, et il faut qu'elles précédent de manière significative ce que la foule va demander. Changer de Premier ministre ou de gouvernement, ou promettre de ne pas se représenter aux élections n'a été suffisant ni pour sauver le président Ben Ali (qui s'est contenté de la seconde promesse) ni pour sauver Moubarak (qui a eu recours aux deux). En essayant de sauver l'essentiel, à savoir rester encore quelques temps au pouvoir, ils ont annoncé des concessions qui ont signalé aux manifestants que l'opportunité politique était devant eux et que de réels changements étaient à leur portée. C'est ainsi qu'au Maroc, par exemple, le roi a surpris les acteurs politiques par l'agenda ambitieux qu'il a proposé dans son discours du 9 mars, et a lancé un processus qui n'a certes pas réussi à neutraliser le mouvement de la rue, mais qui lui a fait concurrence en imposant un agenda tout à la fois positif et parallèle. Ainsi, même si le roi n'a pas totalement réussi à imposer le sien, il a néanmoins réussi à se maintenir dans la dynamique positive des propositions. Il ne

51. Moubarak père préparait la candidature de son fils Gamal pour être son successeur à la tête de l'État. Moubarak fils avait des appuis dans les milieux d'affaire alors que les militaires n’avaient jamais été convaincus de la solidité de sa candidature. 
s'agit pas d'une confrontation violente, mais d'une confrontation entre deux projets.

L'emprise des régimes sur le pouvoir et les instruments et alliances qu'ils utilisent pour s'y maintenir constituent aussi des leçons différentes par rapport aux événements intervenus dans les pays voisins. En effet, les régimes égyptien et algérien ont opté pour des voies distinctes mais pour l'instant tout aussi efficaces dans l'objectif de leur maintien au pouvoir : en Égypte, l'armée a refusé dès le départ de tirer sur la foule et s'est portée garante de la sécurité des manifestants. Elle a fini par faire ce qu'elle aurait préféré ne pas avoir à faire - abandonner Moubarak - mais en veillant à protéger ses propres intérêts et à préserver son rôle dans le système du pouvoir égyptien. Quant à l'Algérie, le régime, et l'armée en particulier, a tout simplement décidé de ne pas autoriser de manifestations à Alger et d'établir un cordon sécuritaire important chaque fois qu'une manifestation populaire était programmée. En parallèle, le président Bouteflika a fait des concessions symboliques, comme le 3 février par exemple, quand il a promis de suspendre l'état d'urgence qui était déclaré depuis février 1992, ou le 15 avril quand il a annoncé une réforme de la Constitution ainsi que la modification de la loi électorale et de la loi sur les partis politiques 52. Cependant Bouteflika n'a pas suspendu la loi de 2001 qui interdit les manifestations dans la capitale Alger et a abusé des largesses que lui accorde la rente pétrolière. En général, les manifestations n’ont pas eu lieu, malgré le succès relatif de la manifestation des étudiants à Alger le mercredi 13 avril, ce qui ne veut certainement pas dire que l'Algérie soit plus stable que les autres pays de la région. C'est donc en ce sens que les événements d'un pays à l'autre s'influencent mutuellement, et que l'idée de réseau d'événements présentée par Tilly est celle qui représente le mieux ce qui s'est passé au sein de ce qu'on appelle le monde arabe.

Ainsi, les régimes syrien, libyen, yéménite, jordanien, algérien et marocain ont tous pu apprendre des leçons des chutes des présidents tunisien et égyptien. Que ce soit en faisant appel à la manière forte et à la répression, ou aux négociations et aux concessions, ils ont réagi de manière différente. L'idée d'événements en réseau s'applique d'ailleurs aux influences à l'œuvre aussi bien parmi les régimes que parmi les manifestants : le succès des Tunisiens et des Égyptiens a donc eu un effet d'entraînement sur plusieurs autres pays arabes. En effet, les conditions de répression, le manque de liberté et les opportunités réduites préexistaient mais un catalyseur était nécessaire pour mener à la révolte. Le catalyseur, dans ce cas précis, a été la conjonction des évènements en Tunisie et en Égypte. Rappelons, à titre d'exemple, le fait que bien des jeunes marocains s'étaient immolés au cours des dernières années, y compris devant le Parlement marocain à Rabat en 2010. Mais aucune de ces

52. «Abdelaziz Bouteflika annonce des réformes politiques », Le Point.fr, 16 avril 2011, (http://www.lepoint.fr/monde/abdelaziz-bouteflika-annonce-des-reformes-politiques-1604-2011-1320059_24.php). 
immolations n'ont eu l'effet de l'immolation et la mort de Bouazizi en Tunisie. Ainsi, la séquence des évènements en Tunisie a eu des conséquences fondamentales dans les autres pays, et l'immolation a acquis un tout autre poids en 2011, quand plusieurs jeunes ont suivi le même chemin en espérant les mêmes conséquences. Un phénomène similaire s'applique aux réactions internationales et aux pressions exercées par l'Occident : les erreurs de la France dans le cas de la Tunisie ont été un encouragement pour que le président Sarkozy agisse rapidement et énergiquement, aussi bien en Libye qu'en Syrie, et le fait d'être intervenu en Libye influence, de différentes manières, les décisions internationales, y compris de la France, des États-Unis et du Conseil de sécurité. Les similitudes entre les répressions syrienne et libyenne constituent un argument en faveur d'une autorisation du Conseil de sécurité pour une intervention en Syrie, alors que le fait d'être déjà présents en Libye (en plus de l'Irak et de l'Afghanistan entre autres) représente un handicap pour certains pays occidentaux en vue d'une éventuelle intervention militaire, même sur des bases humanitaires, en Syrie.

\section{Conclusion}

Les révoltes populaires dans les pays arabes nous permettent d'apporter quelques précisions aux modèles qui analysent les mobilisations contre les régimes autoritaires. Tout d'abord, dans le monde arabe, en 2011, les révoltes ont souvent eu lieu dans des pays où il n'y avait pas eu de réformes préalables, et dans lesquels l'environnement politique était aussi clos en 2011 que pendant toute la décennie précédente. Ce que beaucoup de manifestants dans les rues arabes disaient était «kafa» (ça suffit), car ils ne supportaient plus leurs conditions de vie, que ce soit d'un point de vue politique, économique ou social. À l'image des individus qui risquent leurs vies dans de longs et périlleux trajets pour immigrer illégalement en Europe occidentale ou aux ÉtatsUnis car ils ont très peu à perdre, certaines populations arabes (comme en Tunisie, en Syrie et en Libye) sont sorties dans les rues à leurs risques et périls pour essayer de changer de régime. Si Tilly nous dit qu'en Europe de l'Est, en 1989, ce sont les réformes des régimes tout au long des années précédentes qui ont encouragé l'émergence de revendications, dans plusieurs pays arabes, il ne s'agissait ni de cela, ni de groupes d'opposants organisés. Ce sont plutôt des individus et des groupes d'individus peu ou pas organisés initialement, mais qui partageaient les mêmes frustrations, les mêmes conditions de vie et les mêmes types de bourreaux. Par contre, une fois engagés sur la voie de la protestation, ils n'ont plus reculé, à l'image des tunisiens qui, après la chute de Ben Ali, ont fait chuter deux gouvernements de transition, ou les Égyptiens qui sont revenus pour occuper la place Tahrir quand ils ont senti leur révolution en péril, ou bien encore les Syriens qui ont essuyé des tirs à balles réelles dès leurs premières manifestations, sans pour autant avoir jamais reculé. Le slogan marocain «mamfakinch» (ce n'est pas acceptable) représente bien cette attitude déterminée des jeunes manifestants des pays arabes en 2011. 
Ainsi, si parler de structures d'opportunités politiques indique une inclinaison vers la structure au détriment de l'acteur, les révoltes de 2011 dans le monde arabe ont souligné le rôle central que les acteurs peuvent jouer dans les mobilisations, obligeant à une certaine adaptation du modèle. Les tunisiens n'avaient pas connu de réformes dans leur système politique national et les manifestants ont dû forcer de nouvelles voies et créer de nouveaux liens ainsi que de nouveaux espaces là où il n'y en avait pas. Et si en Égypte ou au Maroc, quelques réformes avaient été entreprises, ce n'est pas pour autant que les choix des acteurs, aussi bien les manifestants que les régimes en place, n'ont pas joué un rôle important dans l'évolution qu'ont connue ces pays en 2011.

Il faut néanmoins redéfinir ce qu'on entend par réformes politiques. Comme suggéré ci-dessus, dans le monde arabe, l'extension de la participation politique ne peut être comprise que si on y inclut d'autres formes (quasi) politiques de participation dans la vie publique. Ainsi, alors que partis et syndicats ont vu leurs espaces réduits, d'autres acteurs ont dû intervenir et combler le vide public. Les ouvertures et les opportunités qui se sont donc présentées n'étaient pas exclusivement politiques, mais avaient plutôt trait à l'espace public en général.

Par contre, le succès de la révolte tunisienne a créé de nouvelles opportunités politiques dans le reste des pays arabes, en forçant aussi bien les régimes que les puissances occidentales à réagir. Ainsi, le facteur externe convoqué par Skocpol s'est matérialisé pour les autres pays arabes, sans s'être manifesté pour les Tunisiens. Mais dans le cas de ces autres pays arabes, il faut inclure deux nouvelles dimensions, une temporelle et l'autre internationale : la fragilisation des régimes arabes durant l'année 2011 a été initialement externe, et l'opportunité politique a été de nature conjoncturelle et non pas structurelle.

Ainsi, pour comprendre les révoltes arabes en 2011, il faut analyser les ouvertures sociales, culturelles, économiques et, à un moindre égard, politiques, qui ont eu lieu dans ces sociétés au cours des années précédentes pour percevoir les opportunités qui se sont présentées aux populations arabes. Il faut aussi ajouter une dimension temporelle, de processus à court terme, ainsi qu'une dimension internationale, pour pouvoir inclure les différentes dynamiques qui se sont imposées aux régimes. Cette dynamique est somme toute conjoncturelle, plutôt que structurelle, et elle repose sur les choix et les options que les acteurs retiennent. 